\title{
A BeppoSAX view of the Centaurus Cluster
}

\author{
S. Molendi ${ }^{1}$, S. De Grandi ${ }^{2}$, and M. Guainazzi ${ }^{3}$ \\ 1 Istituto di Fisica Cosmica, CNR, via Bassini 15, 20133 Milano, Italy \\ 2 Osservatorio Astronomico di Brera, via Bianchi 46, 23807 Merate (LC), Italy \\ 3 XMM-Newton SOC, VILSPA ESA, Apartado 50727, 28080 Madrid, Spain
}

Received 4 October 2001 / Accepted 6 May 2002

\begin{abstract}
We present results from the analysis of a BeppoSAX observation of the Centaurus Cluster. The radial metal abundance profile shows evidence of a large enhancement in the core, where $\mathrm{Ab}>1$ (sol. units). The temperature map indicates that the cluster is characterized by a gradient oriented in the NW/SE direction, with cooler gas in the NW and hotter gas in the SE $(\Delta k T \sim 1 \mathrm{keV})$. In hard X-rays, where the PDS detects emission with a flux of $2.2 \times 10^{-11} \mathrm{erg} / \mathrm{cm}^{2} / \mathrm{s}$ in the $20-200 \mathrm{keV}$ band, the spectrum lies above the extrapolation of the cluster thermal emission. We discuss several possible interpretations for the hard excess finding that none is completely satisfactory.
\end{abstract}

Key words. X-rays: galaxies: clusters - general: individual: Centaurus

\section{Introduction}

The Centaurus Cluster is amongst the nearest $(z=0.0114)$ and brightest clusters in the X-ray sky. It has been looked at with all major X-ray missions. In recent years, observations with the ASCA satellite have produced a number of interesting results. Analysis of the core of the cluster has provided evidence for a high metal concentration, the measured abundance exceeds the solar value (Fukazawa et al. 1994), and for a cool emission component (Fukazawa et al. 1994; Finoguenov et al. 2001; Furusho et al. 2001). Churazov et al. (1999) and Furusho et al. (2001) have detected asymmetric temperature variations on the few hundred kpc scales. The fact that the hot region located SE of the cluster is roughly coincident with the position of NGC 4709, which is the dominant galaxy of the Cent45 subcluster, indicates that the cluster is probably undergoing a merger event. In this paper we present results from the analysis of a BeppoSAX (Boella et al. 1997a) observation of the Centaurus cluster. We use the MECS experiment (Boella et al. 1997b), which is characterized by a spatial resolution a factor of $\sim 2$ better than that of the ASCA instruments, to investigate the temperature and metal abundance structure of Centaurus. We also use the PDS experiment (Frontera et al. 1997), to study the hard X-ray emission. The paper is organized as follows: in Sect. 2 we describe the data reduction. In Sect. 3 we present the spatially resolved spectroscopy performed with the MECS instrument. In Sect. 4 we describe the PDS spectrum. In Sect. 5 we discuss our results and compare them to previous findings. Confidence intervals are at the $68 \%$ level for

Send offprint requests to: S. Molendi, e-mail: silvano@ifctr.mi.cnr.it one interesting parameter, unless otherwise stated. We assume $H_{0}=50 \mathrm{~km} \mathrm{~s}^{-1} \mathrm{Mpc}^{-1}$.

\section{Observations and data preparation}

BeppoSAX observed the Centaurus Cluster between 2000 July 3 (15:03:31 UTC) and July 5 (11:21:44 UTC). All the instruments were operated in standard photon-counting direct modes, the exposure times were respectively $70 \mathrm{ks}$ for MECS and $31 \mathrm{ks}$ for PDS. Data reduction followed standard procedures as described, e.g., in Guainazzi et al. (1999). In this paper only data from the Medium Energy Concentrator Spectrometer (MECS, 1.8-10.5 keV) and from the Phoswitch detector System (PDS, 13-200 keV) will be presented.

We have analyzed the data from the MECS2 and MECS3 separately. The SAXDAS package under FTOOLS environment has been applied to produce equalized and linearized MECS event files. Using the information contained in the housekeeping files we have rejected all events which have occurred at times when the instantaneous pointing direction differed by more than $10^{\prime \prime}$ from the mean pointing direction.

The PDS data have been reduced using a variable Rise Time threshold method, which allows an increase of the signal-tonoise $(S / N)$ ratio for sources fainter than $1 \mathrm{mCrab}$. The corresponding normalization factor (0.8; Fiore et al. 1999) has been applied in all the spectral fits discussed in this paper.

\section{Spatially resolved spectroscopy}

Spectra have been prepared for analysis following the guidelines described in De Grandi \& Molendi (2001) and 
De Grandi \& Molendi (2002), below we summarize the various operations which have been performed.

Spectral distorsions introduced by the energy dependent PSF are taken into account using the EFFAREA program publicly available within the latest SAXDAS release.

The temperature and metal abundance radial profiles are truncated at the last bin where the source counts exceed $30 \%$ and $40 \%$ respectively of the total (i.e. source+background) counts. The energy range used for the spectral fitting is 2$10 \mathrm{keV}$ with the following exceptions. In the outermost bins, if the source counts drop to less than $50 \%$ with respect to the total counts, we restrict the energy range to $2-8 \mathrm{keV}$, to avoid possible distortions from the hard MECS instrumental background. A strongback correction has been applied to the effective area for the $8^{\prime}-12^{\prime}$ annulus, in this bin we restrict our analysis to the range $3.5-10 \mathrm{keV}$ to avoid the low energy part of the spectrum where our correction is less reliable. All other regions of the detector covered by the strongback have been appropriately masked and the data rejected.

The background subtraction has been performed using spectra extracted from blank sky events files in the same region of the detector as the source.

\subsection{Radial profiles}

The cluster has been divided into concentric annuli centered on the X-ray emission peak. Out to $8^{\prime}$ we accumulated spectra from 4 annular regions each $2^{\prime}$ wide, beyond this radius we accumulated spectra from annuli $4^{\prime}$ wide.

We have fitted each spectrum with a single temperature model (MEKAL code in XSPEC) absorbed by the galactic line of sight hydrogen column density, $N_{\mathrm{H}}$, of $8.06 \times 10^{20} \mathrm{~cm}^{-2} \mathrm{de}-$ rived from HI maps (Dickey \& Lockman 1990). All spectra, with the exception of the one associated to the innermost bin, can be adequately fit with the above model. In the innermost region the single temperature model leaves a rather large excess above $\sim 5 \mathrm{keV}$. Since ASCA (e.g. Makishima et al. 2001) and XMM-Newton (Molendi \& Pizzolato 2001) studies of the cores of centrally peaked clusters have shown that the spectra accumulated from these regions are generally better fitted by multi temperature models than by single temperature models, we have performed a new fit with a model including a second MEKAL component. The metal abundace of this second component has been linked to the metal abundance of the first. Since the temperature of the hotter component is not well constrained we have fixed it to $4 \mathrm{keV}$, which is approximatively the largest temperature we measure in our profile (see Fig. 1) and in rough agreement with a maximum temperature of $4.4 \mathrm{keV}$ found by Ikebe et al. (1999) from the analysis of ASCA data. The two temperature model yields a substantially better fit than the one temperature model, the improvement is statistically significant at more than the $99 \%$ level according to the F-test. Note however that the two temperature modeling should be regarded as a rough attempt to reproduce the complex spectrum resulting from the superposition of the cooler emission coming from the core of the cluster with the hotter emission coming from regions further out, which happen to be on the same line of sight.

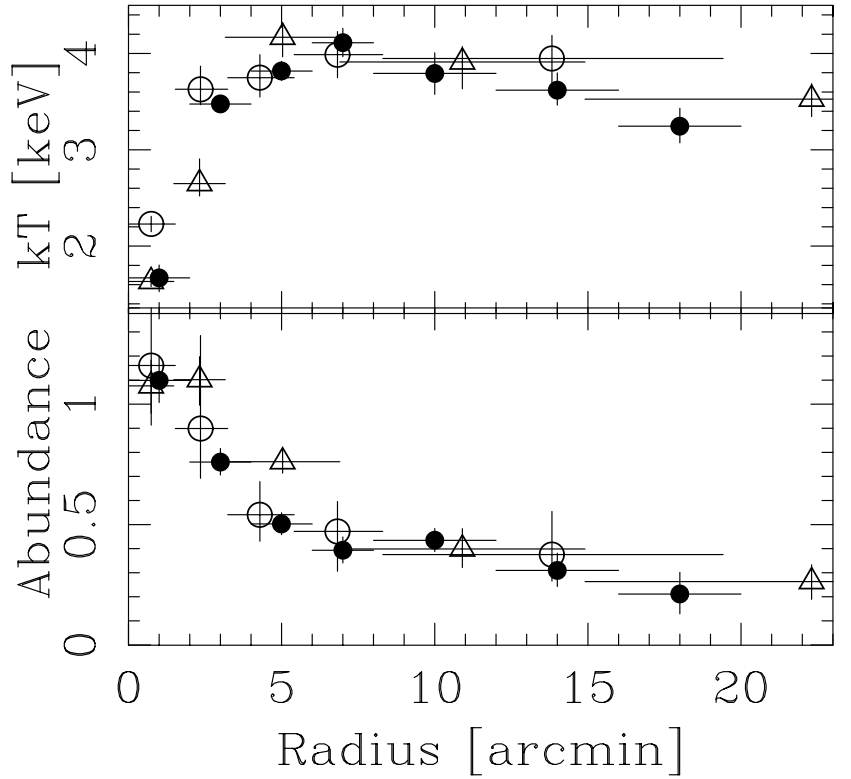

Fig. 1. Top panel: projected radial temperature profile for the Centaurus Cluster. Filled circles indicate temperatures obtained from BeppoSAX MECS data. Open circles and open triangles represent the temperature profile derived from ASCA data respectively by White (2000) and by Finoguenov et al. (2001). Bottom panel: projected radial abundance profile. Symbols as for Top Panel.

Given the limitations of the MECS instrument both in terms of spatial resolution and of band coverage, the MECS data does not allow a more detailed analysis of the temperature structure in the core of Centaurus.

In Fig. 1 we show the temperature and abundance profiles obtained from the spectral fits with single temperature models. The values reported for the innermost bin are respectively the lower temperature and the metal abundance obtained with the 2 temperature model discussed above.

The temperature profile in this, as in other centrally peaked clusters, is found to rise with increasing radius, roughly leveling off at the cooling radius, $r_{\mathrm{c}} \sim 4^{\prime}$ (Peres et al. 1998). Beyond $r_{\mathrm{c}}$ the profile shows a drop. When fitting data for $r>4 \mathrm{arcmin}$, a declining line gives a slightly better fit than a constant (the improvement is significant only at the $75 \%$ level according to the F-test). Note however that if we concentrate only on the last 4 data points ( $r>6$ arcmin) the improvement is found to be significant at more than the $99 \%$ level.

The metal abundance profile shows a striking excess in the innermost bin. We remark that the measurement in this bin is not particularly sensitive to the spectral model, indeed the single temperature and 2 temperature models described above give very similar results. Metal abundance excesses in the core of so-called cooling-flow clusters have been found in virtually all BeppoSAX observations (see De Grandi \& Molendi 2001), however the value we find for Centaurus is the largest measured so far and the only one to exceed the solar value. The abundace drops rapidly with increasing radius reaching a value of $\sim 0.4$ solar units at $\sim 7^{\prime}$ from the center. From here on the abundance declines gently, reaching a value of $\sim 0.2$ solar units at $\sim 18^{\prime}$ from the center. While the abundance excess in 


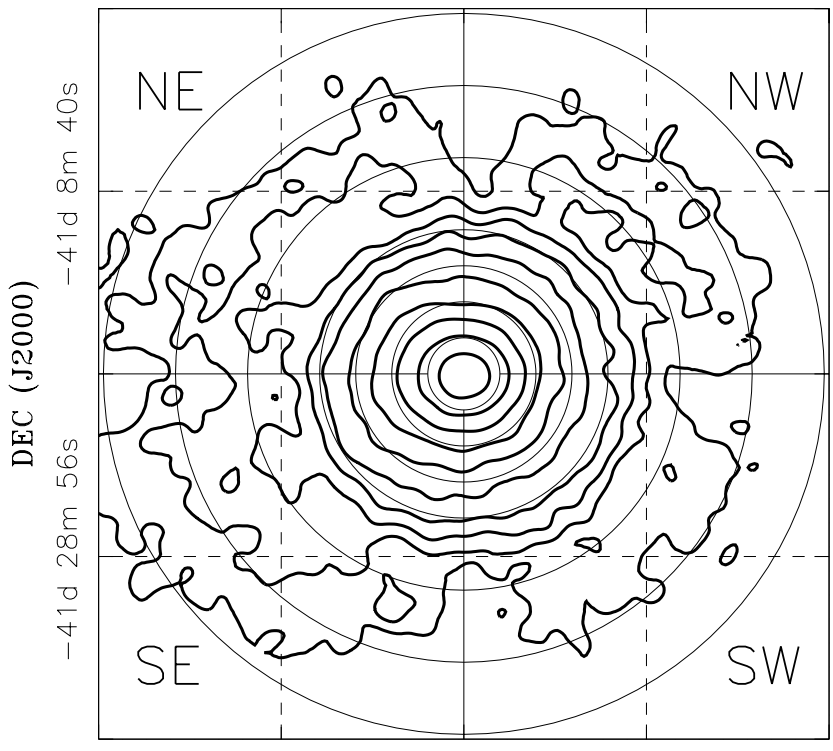

$12 \mathrm{~h} 49 \mathrm{~m} \mathrm{42s}$

RA (J2000)

Fig. 2. BeppoSAX MECS image of the Centaurus Cluster. Contour levels are indicated by the thick lines. The thin lines show how the cluster has been divided to obtain temperature and abundance maps. Note that no correction for the strongback has been applied to the image.

the core is most likely associated to enrichment from the $\mathrm{cD}$ galaxy NGC 4696 itself, the gentle declining profile observed further out could be due to enrichment by galaxies in the core of Centaurus similar to what has been found by Ezawa et al. (1997) in the case of AWM 7.

\subsection{Maps}

As shown in Fig. 2, we have divided the MECS image of the Centaurus cluster into 4 sectors: NW, SW, SE and NE, each sector has been divided into 6 annuli with bounding radii, $2^{\prime}-$ $4^{\prime}, 4^{\prime}-6^{\prime}, 6^{\prime}-8^{\prime}, 8^{\prime}-12^{\prime}, 12^{\prime}-16^{\prime}$ and $16^{\prime}-20^{\prime}$. In Figs. 3 and 4 we show respectively the temperature and abundance profiles obtained from the spectral fits for each of the 4 sectors. Note that the abundance profiles extend only to $16^{\prime}$ because some of the spectra in the $16^{\prime}-20^{\prime}$ annulus do not meet the more stringent conditions required to accept an abundance measurement. To ease the comparison between measurements in different sectors we have drawn a constant line for a temperature of $4 \mathrm{keV}$ and a metal abundance of 0.5 in Figs. 3 and 4 respectively.

In Table 1 we report the best fitting constant temperatures and abundances for the profiles shown in Figs. 3 and 4.

The mean temperature of the NW sector is the smallest, $3.2 \pm 0.1 \mathrm{keV}$, while that of the SE is the highest, $4.0 \pm 0.1 \mathrm{keV}$. The difference in these values is significant at more than the $5 \sigma$ level indicating that the cluster is characterized by an azimuthal temperature gradient. There appears to be a cooler region NW and a hotter region SE of the center. A fit to the temperatures of the 4 sectors in the annulus with bounding radii $2^{\prime}-4^{\prime}$ with a constant, yields $\chi^{2}=20.1$ for 3 d.o.f., with an associated probability for the temperature to be constant of $1.6 \times 10^{-4}$,

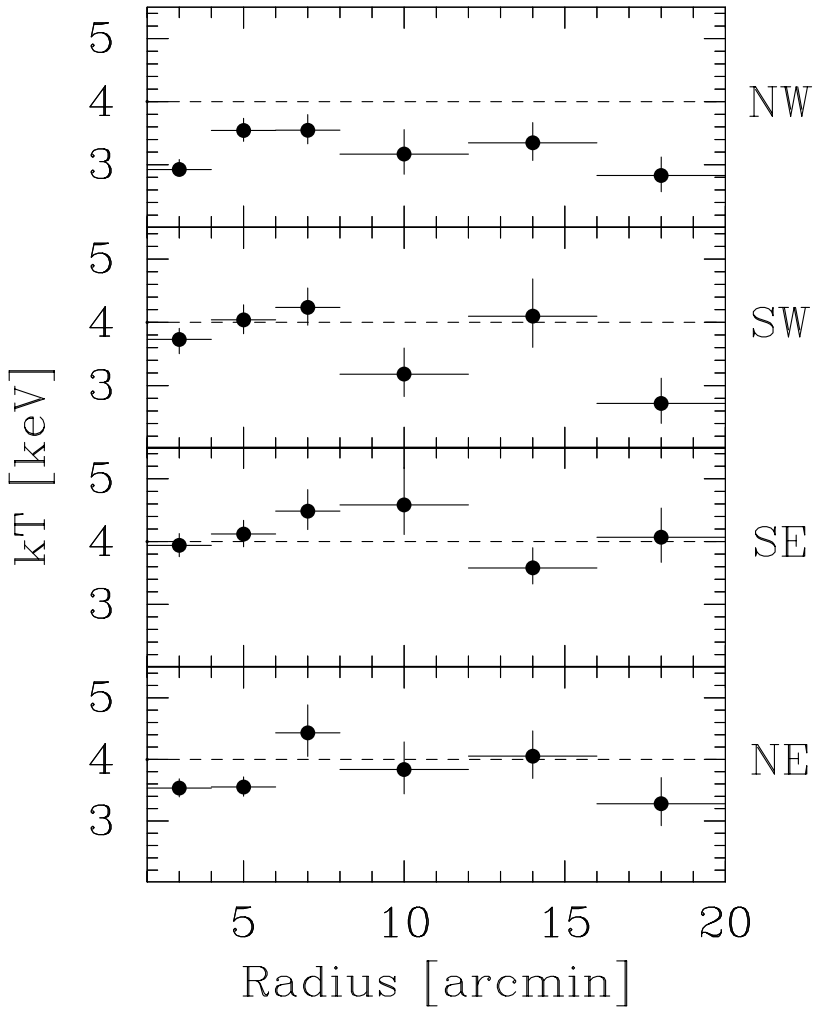

Fig. 3. Radial temperature profiles for the NW sector (first panel), the SW sector (second panel), the SE sector (third panel) and the NE sector (forth panel). The dashed horizontal lines at $4 \mathrm{keV}$ are drawn to ease the comparison between different sectors.

indicating that the azimuthal temperature gradient extends to the smallest scales accessible to the MECS. The NW and the SE sectors are again respectively the coolest and the hottest ones.

Our data does not show compelling evidence for azimuthal abundance gradients. There is however a hint of an abundance excess in the NW sector of the $2^{\prime}-4^{\prime}$ annulus, which has an abundance larger than those of all other sectors of the same annulus and comparable to that of the $0^{\prime}-2^{\prime}$ annulus. Interestingly this is also the sector showing the smallest temperature in the $2^{\prime}-4^{\prime}$ annulus.

\section{The hard X-ray spectrum}

In the $\left(1.3^{\circ}\right)^{2}$ PDS field-of-view a signal at about $3.6 \sigma$ level was detected. In the $13-200 \mathrm{keV}$ energy band the net background-subtracted count rate is $0.161 \pm 0.044$. The error includes the typical systematic uncertainties on the PDS background subtraction algorithm (see the discussion in Matt et al. 1997). A simple power-law fit yields a $\Gamma=1.5 \pm_{0.8}^{1.4}$ $\left(\chi^{2}=15.1 / 22\right.$ d.o.f.). A fit with a bremsstrahlung model is equally good, but only a $90 \%$ lower limit of $30 \mathrm{keV}$ on the electron temperature can be set. The observed 20-200 keV flux is $2.2 \times 10^{-11} \mathrm{erg} \mathrm{cm}^{-2} \mathrm{~s}^{-1}$, corresponding to a rest-frame luminosity of $9.4 \times 10^{42} \mathrm{erg} \mathrm{s}^{-1}$ at the redshift of NGC 4696 .

The extrapolated flux in the $2-10 \mathrm{keV}$ band is $4.2 \times$ $10^{-12} \mathrm{erg} \mathrm{cm}^{-2} \mathrm{~s}^{-1}$. The probability that the PDS detection is due to a serendipitous source in the PDS field of view is $\sim 5 \%$, 
Table 1. Best fit constant values for the temperature and the abundance for the azimuthally averaged radial profiles (All) and for the profiles of the 4 sectors (NW, SW, SE and NE).

\begin{tabular}{lcrccrc}
\hline \hline Sector & $\begin{array}{c}k T \\
(\mathrm{keV})\end{array}$ & $\chi^{2}$ /d.o.f. & Prob. $^{a}$ & $\begin{array}{c}\text { Abundance } \\
(\text { Solar Units })\end{array}$ & $\chi^{2} /$ d.o.f. & Prob. $^{a}$ \\
\hline \hline All & $3.6 \pm 0.1$ & $23.2 / 5$ & $3.1 \times 10^{-4}$ & $0.47 \pm 0.02$ & $42.6 / 5$ & $4.5 \times 10^{-8}$ \\
\hline NW & $3.2 \pm 0.1$ & $10.0 / 5$ & $7.5 \times 10^{-2}$ & $0.40 \pm 0.05$ & $25.6 / 4$ & $3.9 \times 10^{-5}$ \\
SW & $3.8 \pm 0.1$ & $13.0 / 5$ & $2.4 \times 10^{-2}$ & $0.47 \pm 0.05$ & $6.5 / 4$ & $1.7 \times 10^{-1}$ \\
SE & $4.0 \pm 0.1$ & $5.0 / 5$ & $4.2 \times 10^{-1}$ & $0.46 \pm 0.04$ & $6.8 / 4$ & $1.4 \times 10^{-1}$ \\
NE & $3.6 \pm 0.1$ & $5.7 / 5$ & $3.4 \times 10^{-1}$ & $0.57 \pm 0.05$ & $6.0 / 4$ & $2.3 \times 10^{-1}$ \\
\hline \hline
\end{tabular}

${ }^{a}$ Probability for the observed distribution to be drawn from a constant parent distribution.

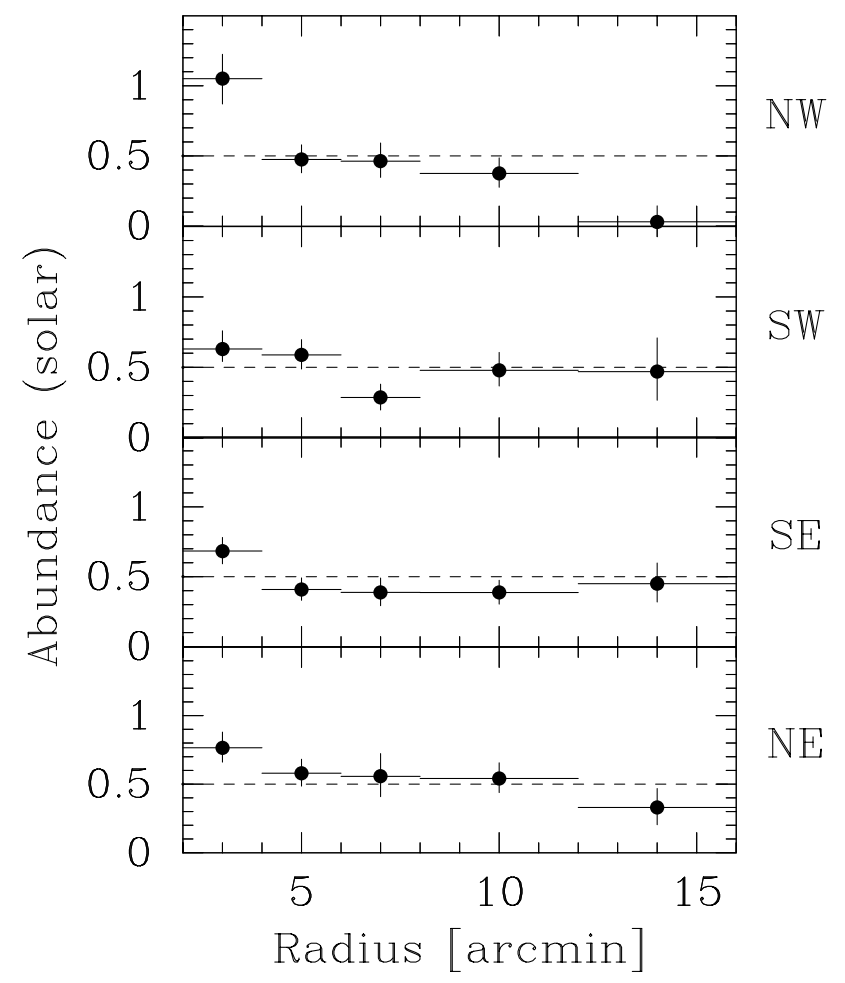

Fig. 4. Radial abundance profiles for the NW sector (first panel), the SW sector (second panel), the SE sector (third panel) and the NE sector (forth panel). The dashed horizontal lines at 0.5 solar units are drawn to ease the comparison between different sectors.

according to the Cagnoni et al. (1998) $\log N-\log S$. This is actually a lower limit, given the enhanced density of AGN expected in the cluster environment. A more accurate estimation, which takes into account the $\log N-\log S$ of the Centaurus Cluster, is discussed in Sect. 5. The PDS data points lie well above the extrapolation of the MECS thermal best-fits for all the annuli. The PDS emission could be due to diffuse emission related to the merger in Centaurus, however the absence of a radio halo argues against this possibility. Alternatively the PDS excess could come from a hard point-like source, which is either unresolved by the MECS, or lies beyond the MECS field of view (we recall that the MECS has a circular FOV with a radius of $\sim 22 \mathrm{arcmin}$ ). In order to investigate the point source alternative, we have retrieved from the public archive the data of a Chandra observation of NGC 4696, performed on
May 22, 2000. The ACIS-S was operated in the standard configuration, being exposed for a total exposure time of 32.1 ks. The Chandra observation covers, however, only a small part of the PDS field-of-view, the ACIS-S FOV is a square 8 arcmin on the side. We have extracted an image of the whole Chandra field from the "level 2" event list. We employed the energy band between 5 and $10 \mathrm{keV}$ to minimize the contribution of the cluster emission and therefore optimize the detection likelihood for point sources. The brightest detected point source has coordinates: $\alpha_{2000}=12^{\mathrm{h}} 49^{\mathrm{m}} 06^{\mathrm{s}} .2$, $\delta_{2000}=-41^{\circ} 17^{\prime} 52^{\prime \prime}$, and net count rate of $(6.8 \pm 1.9) \times 10^{-4} \mathrm{~s}^{-1}$. The extrapolation of this rate into the PDS energy bandpass, assuming a $\Gamma=1.5$ power-law, is $4 \times 10^{-3} \mathrm{~s}^{-1}$ therefore about 40 times lower than the PDS count rate measured during the BeppoSAX observation. The Chandra count rate 3- $\sigma$ upper limit at the position of the optical nucleus of NGC 4696 is $9 \times 10^{-5} \mathrm{~s}^{-1}$. If the detection in the PDS is due to the emission of a strongly absorbed active nucleus at the position of the optical nucleus, the column density, $N_{\mathrm{H} \text {,nuc }}$ must be $\simeq 3.3 \times 10^{24} \mathrm{~cm}^{-2}$.

\section{Discussion}

In Fig. 1 we have compared our radial temperature and abundance profiles with temperature and abundance profiles derived from ASCA data independently by White (2000) and Finoguenov et al. (2001). Overall the BeppoSAX temperature profile is in good agreement with the ASCA profiles. There are some differences within the innermost 4 arcmin amongs the three profiles. Such differences are likely related to the different spectral bandpass over which the spectral fitting of the data has been performed. At radii larger than $4^{\prime}$ the three different temperature measurements yield results which are comfortably consistent. The abundace profiles are also in good agreement. There is one apparent exception, i.e. the abundace measurement at $5^{\prime}$ by Figuenov et al. (2001). The difference here however is due to the fact that the bin adopted by Finoguenov et al. (2001) is substantially larger than those used in White (2000) and in our own analysis. Thus the spectrum fitted by Finoguenov et al. (2001) also includes emission from further in where the abundance is larger.

Our temperature map shows evidence of a gradient in the $\mathrm{NW} / \mathrm{SE}$ direction, the cooler gas is in the NW sector and the hotter gas in the SE. Interestingly the ASCA temperature maps of Centaurus reported by Churazov et al. (1999) and 
Furusho et al. (2001) show evidence of a temperature gradient oriented as ours.

We present here an abundance map for Centaurus. It does not provide compelling evidence for azimuthal abundance gradients. The only result of some interest is the hint of an excess in the NW sector of the $2^{\prime}-4^{\prime}$ annulus. Interestingly this sector is also characterized by the smallest temperature in the $2^{\prime}-4^{\prime}$. Thus both in terms of temperature and of metal abundance the emission in this sector is more similar to the emission from the innermost region than to that of the other sectors of the same annulus. It is tempting to speculate that gas in this sector may be more closely related to gas in the innermost region than to gas at similar radial distances in other sectors.

Shortly after this paper was submitted, Sanders et al. (2002) published a detailed analysis of the Chandra observation of the Centaurus core. Thanks to its better spatial resolution Chandra can investigate temperature and abundance variations on scales smaller than those accesible to the BeppoSAX MECS. It is therefore quite reasuring that the presence a of cooler and metal richer region extending out to $\sim 3$ arcmin $\mathrm{NW}$ of the core is confirmed by Chandra. Indeed it proves that the MECS are capable of detecting variations in temperature and abundance down to scales of a few arcmin.

Apparently unrelated to the thermal emission imaged by the MECS, a signal at the $3.6 \sigma$ level is measured in the 13 $200 \mathrm{keV}$ energy band with the PDS instrument. It is impossible from the PDS data alone to establish the origin of this emission. A possible explanation could be that we are observing diffuse hard X-ray emission associated to the cluster merger, however, the fact that no radio halo has been detected in the Centaurus cluster, argues against this possibility.

The X-ray luminosity associated to the PDS detection exceeds $10^{43} \mathrm{erg} \mathrm{s}^{-1}$. This would favor emission from a strongly absorbed active nucleus. If this hypothesis is true, the comparison with a non simultaneous Chandra observation constraint the nuclear absorbing column density to be $\simeq 3.3 \times 10^{24} \mathrm{~cm}^{-2}$. This hypothesis is, however, not supported by evidences in other wavelength. No highly ionized emission lines has ever been discovered in optical spectroscopy of the NGC $4696 \mathrm{nu}-$ cleus. Although this is not a conclusive proof (other cases of optically "dull" AGN are known and being discovered, the prototype of this class being NGC 4945; Matt et al. 2000; see also the discussion in Mushotzky et al. 2000), there is no wavelength in the NGC 4696 spectral energy distribution where the reprocessed X-rays from the absorbed active nucleus seem to emerge. The total far infrared $(40-120 \mu \mathrm{m})$ luminosity is $\sim 1.2 \times 10^{42} \mathrm{erg} \mathrm{s}^{-1}$ only (de Jong et al. 1990). The IR colors as measured by IRAS are the coolest among the elliptical galaxies of the Jura et al. (1987) sample. The possibility of a serendipitous source in the PDS cannot be excluded a priori, given the lack of simultaneous imaging coverage of its field of view. We have tried to estimate the likelihood of such an event using the ROSAT All Sky Survey (RASS). The extrapolation of the PDS spectrum in the ROSAT PSPC band yields a count rate of $0.12 \mathrm{cts} \mathrm{s}^{-1}$. Within the PDS field of view, no RASS source is detected at this intensity level. However, X-ray absorbed sources could be easily missed in the ROSAT Centaurus Cluster census. One can estimate the expected density of all X-ray sources in Centaurus by looking at a wider area to increase the counting statistics, and correcting the results for the fraction of missed absorbed objects. In a $3^{\circ}$ radius circular area, 6 sources brighter than the above PSPC count rate limit are detected, corresponding to about 1.1 source per $\left(1.3^{\circ}\right)^{2}$ circle, even if no correction for the obscured objects is done. Of course, the extrapolation of the X-ray counts along the almost two order of magnitudes in energy which separate the energy bandpasses of the BeppoSAX/PDS and the ROSAT/PSPC introduces rather large uncertainties in this estimate. Alternatively, one may employ the $\log N-\log S$ of the Centaurus Cluster (Jerjen \& Dressler 1997) to estimate the number of potential AGN fields. The PDS measurement corresponds to a $1 \mathrm{keV}$ flux density of $500 \mathrm{mJy}$. Assuming a typical quasar optical-to-X-ray luminosity ratio of $0.5 \mathrm{dex}$ (Elvis et al. 1994), this flux density corresponds to a $B$ magnitude of 10.6. The number of galaxies with $m_{B} \gtrsim 10.6$ in the 2.2 square degrees survey of Jerjen \& Dressler (1997) is $\simeq 12$. Even assuming a $10 \%$ fraction of active galaxies, this implies that the number of expected AGN in the PDS field of view is $\simeq 0.4$. Both the above tests suggest that the PDS detection may be due to a serendipitous X-ray hard field AGN.

Acknowledgements. The BeppoSAX satellite is a joint Italian-Dutch program.

\section{References}

Boella, G., Butler, R. C., Perola, G. C., et al. 1997a, A\&AS, 122, 299 Boella, G., Chiappetti, L., Conti, G., et al. 1997b, A\&AS, 122, 327

Cagnoni, I., della Ceca, R., \& Maccacaro, T. 1998, ApJ, 493, 54

Churazov, E., Gilfanov, M., Forman, W., \& Jones, C. 1999, ApJ, 520, 105

De Grandi, S., \& Molendi, S. 2001, ApJ, 551, 153

De Grandi, S., \& Molendi, S. 2002, ApJ, 567, 163

de Jong, T., Norgaard-Nielsen, H. U., Jorgensen, H. E., \& Hansen, L. 1990, A\&A, 232, 317

Dickey, J. M., \& Lockman, F. J. 1990, ARA\&A, 28, 215

Elvis, M., Wilkes, B. J., McDowell, J. C., et al. 1994, ApJS, 95, 1

Ezawa, H., Fukazawa, Y., Makishima, K., et al. 1997, ApJ, 490, L33

Finoguenov, A., Arnaud, M., \& David, L. P. 2001, ApJ, 555, 191

Fiore, F., Guainazzi, M., \& Grandi, P., Cookbook of BeppoSAX SDC data analysis (Roma: BeppoSAX Science Data Center)

Frontera, F., Costa, E., Dal Fiume, D., et al. 1997, A\&A, 122, 357

Furusho, T., Yamasaki, N. Y., Ohashi, T., et al. 2001, PASJ, 53, 421

Fukazawa, Y., Ohashi, T., Fabian, A. C., et al. 1994, PASJ, 46, L55

Guainazzi, M., \& Matteuzzi, M. 1997, SDC-TR-014 (Roma: BeppoSAX Science Data Center)

Ikebe, Y., Makishima, K., \& Fukazawa, Y., et al. 1999, ApJ, 525, 58

Jerjen, H., \& Dressler, A. 1997, A\&AS, 124, 1

Jura, M., Kim, D. W., Knapp, G. R., \& Guhathakurta, P. 1987, ApJ, 312,11

Matt, G., Fabian, A. C., Guainazzi, M., et al. 2000, MNRAS, 318, 173

Makishima, K., Ezawa, H., Fukazawa, Y., et al. 2001, PASJ, 53, 401

Matt, G., et al. 1997, A\&A, 325, L13

Molendi, S., \& Pizzolato, F. 2001, ApJ, 560, 194

Mushotzky, R. F., Cowie, L. L., Barger, A. J., \& Arnaud, K. A. 2000, Nature 404, 459

Peres, C. B., Fabian, A. C., Edge, A. C., et al. 1998, MNRAS, 298, 416

Sanders, J. S., \& Fabian, A. C. 2002, MNRAS, 331, 273

White, D. A. 2000, MNRAS, 312, 663 